\title{
Medical long-distance monitoring system based on internet of things
}

\author{
Weiping Zhang ${ }^{1,2}$, Mohit Kumar ${ }^{3}$, Junfeng $Y u^{2}$ and Jingzhi Yang ${ }^{2 *}$
}

\begin{abstract}
To allow doctors to monitor the physical parameters of the patient's body in real time and to understand the changes in the patient's condition in time, the medical remote monitoring system based on the Internet of Things was studied. From the perspective of practical application of hospital wards, a medical health monitoring system was designed with the help of CC2430 microcontroller, human information sensor, and microelectronic and modern wireless communication technology. In addition, a sensor node circuit and a coordinator node circuit for collecting medical signals were designed. Meanwhile, the software of wireless sensor network was designed. Finally, the online debugging of each system module was combined with hardware and software. The experimental result proved that the network node was reliable and the data transmission was accurate. It is concluded that the medical monitoring system basically meets the design requirements of this paper.
\end{abstract}

Keywords: Medicine, Remote monitoring, Monitoring system, Sensor, Coordinator

\section{Introduction}

Increasing life expectancy, due to medical progress, and decreasing birth rates lead to a change in the population structure of Germany. As illustrated in Fig. 1, the percentage of people over the age of 65 is steadily increasing while the number of people under the age of 25 is declining. This results in continuously rising costs for healthcare, especially for elderly people. One approach to this problem is the utilization of telemonitoring systems to enable a relocation of the therapy to the patients' home, thus shortening the expensive periods of hospitalization [1].

The Internet of Things (IoT) medical remote monitoring system is to establish a wireless monitoring network in a hospital ward. The patient collects physiological signals by carrying a small sensor node and sends it wirelessly to the user management center. In this way, the patient does not need to connect a variety of wires and even can wear a comfortable electronic fabric. Therefore, they can obtain more free space. This operation is convenient for the diagnosis work. Doctors and nurses can monitor the physiological status of each patient in real time through the user management platform. Finally, doctors' work efficiency is

\footnotetext{
* Correspondence: Iulu1116420@outlook.com

${ }^{2}$ Binhai Industrial Technology Research Institute of Zhejiang University,

Tianjin, China

Full list of author information is available at the end of the article
}

improved, and they have more time to serve patients. Once an emergency occurs, it can be properly handled [2].

In foreign countries, the IoT technology has emerged earlier and its development is relatively mature. In the USA, a number of institutions have launched research on IoT medicine. Intel is currently developing a wireless sensor network system for home care. They embed sensors in shoes, furniture, and home appliances to help older people and people with disabilities live independently. Scientists at the University of Rochester use wireless sensors to create a smart medical room. They use dust to measure important signs of the occupants, sleeping position, and $24 \mathrm{~h}$ of activity every day [3]. In China, the State Key Laboratory of Mobile Communications of Southeast University and China Mobile Jiangsu Corporation jointly developed a real-time health monitoring system to provide real-time health monitoring services for the elderly through the Internet of Things [4]. However, at present, most of researches in China are still focused on theory, and it is rarely used in practice. In the case of IoT medical care, many jobs are just preliminary attempts, or more studies on electronic medical records and single disease detection, and less on in-patient physiological information monitoring. The design of this article applies the Internet of Things to medical monitoring very well. 


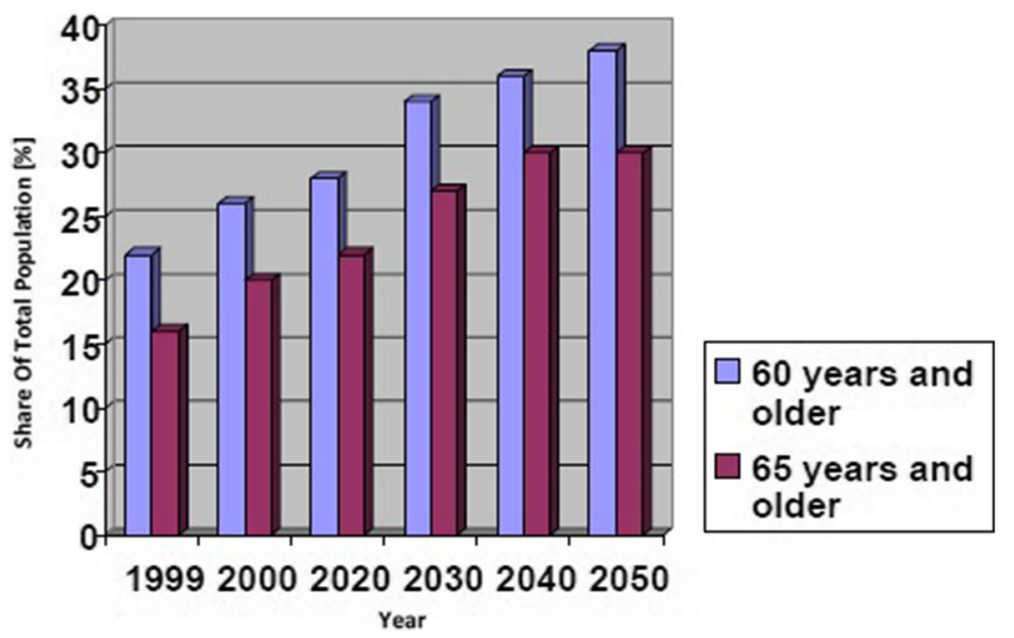

Fig. 1 Projected percentage of elderly people in German population. (Source: German Federal Bureau of Statistics 2010)

\section{Methodology}

\subsection{Overall system design}

Due to limited power, the distance of wireless communication is limited. The ZigBee protocol is used to set up a wireless sensor network to transmit information through wireless communication between a close-range node and a long-distance node. This process is just the same as information transmission between bees [5]. ZigBee technology supports three network topologies, including star-type network, tree-type network, and mesh network. This design selects a tree-type network [6].

The patient carrying the sensor node can move freely within the guardianship. After the sensor acquisition node is started, it applies for joining the tree network and periodically transmits the collected physiological information such as body temperature, pulse, and electrocardiogram wirelessly to the routing node located at the entrance of the ward. The routing node simply processes the collected information and forwards it to the coordinator node. The coordinator node is placed in the doctor's duty room and connected to the PC via a serial port. It is a full-featured device and is the center of the network. It is mainly responsible for establishing, starting up the network, configuring network parameters, gathering the received information, compressing the redundant data, and transmitting useful information to the PC database through the serial port. On-duty doctors can keep track of the patient's physiology through the user management system. In addition, patients who are active outside the hospital's ward can transmit information to the doctor's care unit after joining the network through a sensor node worn by the body. It is shown that the sensor node, the routing node, and the coordinator node make up the wireless sensor network of the ward, and network constitute the entire hospital monitoring network. The monitoring center can also carry out remote data transmission via the Internet and mobile communication networks, sharing information with other monitoring centers and expanding to a telemedicine monitoring network system with wider range. The whole system structure is shown in Fig. 2.

\subsection{System hardware design}

With the rapid development of sensing technology, microelectronics, and information and communication technology, various specialized hybrid integrated circuits have emerged. In general, hybrid integrated circuits can integrate multiple functional modules on a single chip, which greatly improves design efficiency and shortens development cycles. This article uses CC2430 chip, a variety of medical sensors and wireless communication technology to complete the hardware design of medical monitoring system for real-time monitoring of human physiological information, including the circuit design of sensor nodes, routing nodes, and coordinator nodes. The hardware design block diagram of medical monitoring system is shown in Fig. 3.

All three types of nodes use the CC2430 chip as the core controller. The CC2430 integrates an 8051 controller and a 2.4 GHz RF transceiver. It can process, convert, and store physiological parameter information and communicate data wirelessly. The sensor node is responsible for collecting patient physiological signals. It also includes various medical sensor interface circuits and corresponding conditioning circuits. The coordinator node needs to send all the received parameter information to the PC. Therefore, the serial communication module needs to be added.

The routing node is mainly responsible for aggregating the sensor node data and forwarding the data to the coordinator node. As shown in Fig. 4, its hardware structure is basically the same as the coordinator node. It is mainly composed by the CC2430 module, the serial communication module, the LCD display module, and the keyboard module. 


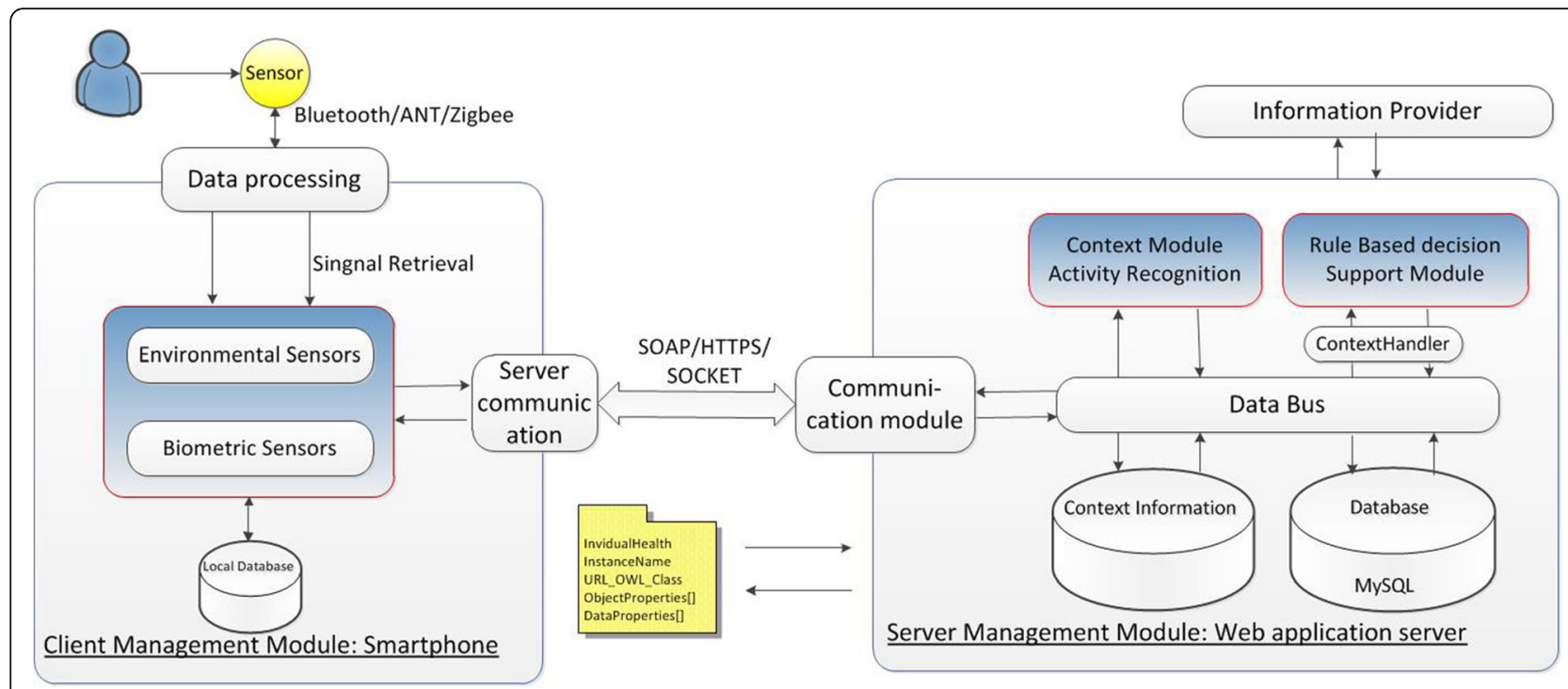

Fig. 2 Structure design of loT based medical remote monitoring system

The sensor node is mainly responsible for collecting the patient's physiological parameter information and periodically sending this information wirelessly to the routing node. Based on the node of the coordinator, the design adds the sensor interface circuit and conditioning circuit and completes the design of the sensor node. The detection of physiological parameters is an important part of the daily examination of inpatients. Many critically ill patients and the elderly also need long-term physical monitoring. The common physiological parameters include body temperature, pulse, blood pressure, heart rate, electrocardiogram (ECG), respiration, oxygen partial pressure, oxygen saturation, and blood glucose. These physiological parameters generally require non-invasive or minimally invasive detection in the monitoring system [7]. The design uses body temperature, pulse, and ECG signals as the collection object. A simple and convenient method of sensor and non-invasive measurement is chosen, and various physiological signal conditioning circuits are designed. Due to content restrictions, only the idea of ECG signal circuit design is listed here.

ECG signals are an important signal for human monitoring. ECG signal is a comprehensive reflection of cardiac electrical activity on the body surface and is of great significance in the diagnosis of heart disease. Electrocardiogram is often used as an aid in clinical disease diagnosis [8]. The ECG acquisition circuit mainly includes preamplifier circuit, $0.05-100 \mathrm{~Hz}$ bandpass filter circuit, $50 \mathrm{~Hz}$ trap circuit, and main amplifier circuit. There are many kinds of interference in ECG measurement, and the most important one is the power frequency $(50 \mathrm{~Hz})$ interference. However, the high common mode rejection ratio of the

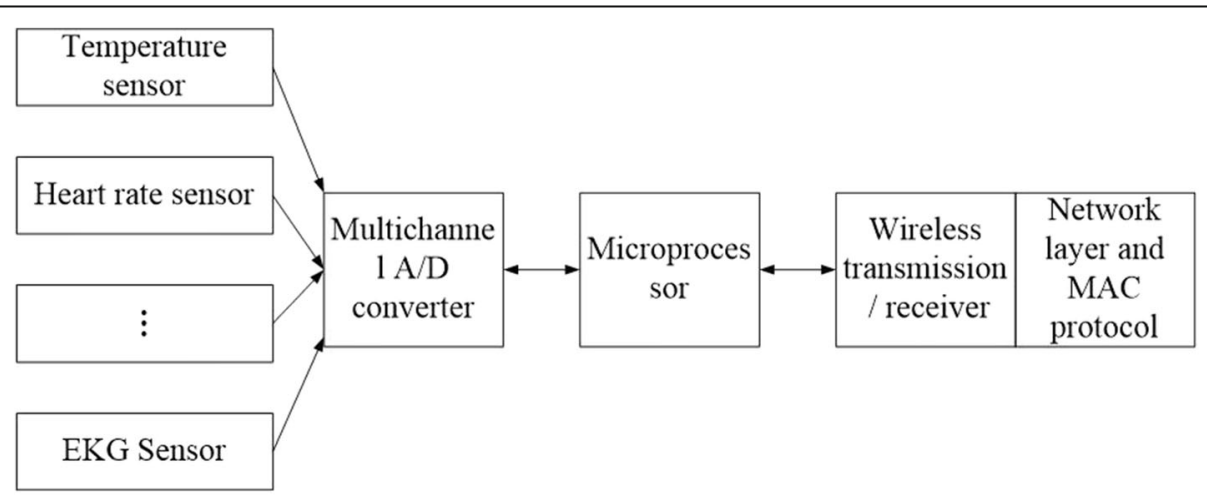

Sensor module

Data processing module
Wireless communication module

Fig. 3 Hardware design block diagram of medical monitoring system 


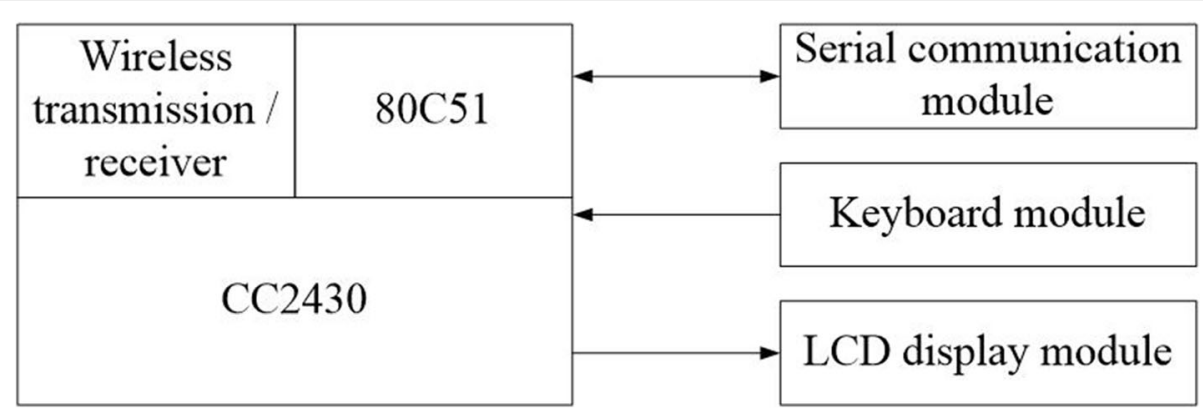

Fig. 4 Coordinator node structure

preamplifier alone is not enough to effectively suppress the power frequency interference, and the driven-right-leg circuit is also needed to eliminate the influence of the power frequency interference. The interference voltage is calculated as:

$$
U_{\text {interfere }}=\frac{U_{\text {space }} \times Z_{2}}{Z_{1}+Z_{2}}=\frac{U_{\text {space }}}{1+\left(Z_{1} / Z_{2}\right)}
$$

The frequency band of human electrocardiographic signals ranges from 0.05 to $100 \mathrm{~Hz}$. During the process of acquiring the ECG signal, the signal will have low-frequency baseline drift due to the patient's activity or device temperature changes, and the ECG signal will not be at the same baseline level. If the drift is too large, it may cause the signal to swing to the VCC or VEE terminal, saturating the amplifier and losing amplification. The high-pass filter circuit can filter out low-frequency components in the input signal and effectively suppress baseline drift. The maximum frequency of the ECG signal is about $100 \mathrm{~Hz}$. To meet the digital signal sampling force, the following formula is obtained:

$$
f_{s} \geq f_{\text {MAX }}
$$

ECG measurement physical diagram is shown in Fig. 5, and hardware node physical diagram is shown in Fig. 6.

\subsection{Algorithm for multivariate medical data modeling}

Data mining techniques such as classification and clustering play a vital role in the development of medical decision support systems contributing to improved healthcare quality. The medical decision-making problems inherently involve complexities and uncertainties, and thus, the researchers have advocated the integration of fuzzy methodologies in medical data interpretation. The handling of uncertainties by capturing of knowledge using fuzzy sets and rules together with an interpretability offered by simple linguistic if-then rules are two most important features of fuzzy methodologies. The fuzzy approaches are commonly applied to medical data classification problems. In our previous work [9], we suggested to represent multi-dimensional medical data by means of an optimal fuzzy membership function, and a carefully designed data model is introduced in a completely deterministic framework where uncertain variables are characterized by fuzzy membership functions.

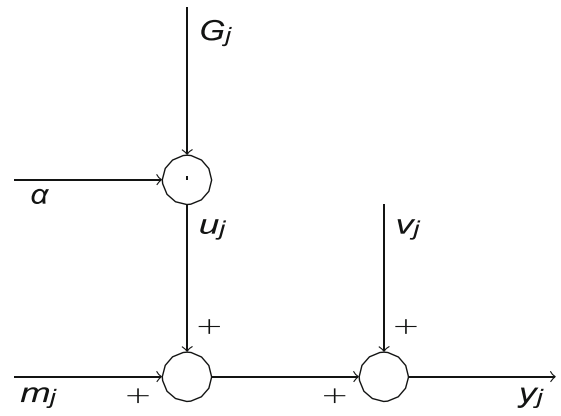

The medical data is multi-dimensional whose good representation by means of fuzzy membership functions is the aim of the mathematical theory presented in this study. This text introduces a data model that takes into account both measurement noise and uncertainties arising from individuality-related factors. A multivariate data sample, represented as $\mathrm{y}=[\mathrm{y} 1 \cdots \mathrm{yP}] \mathrm{T} \in \mathrm{RP}$, is assumed to be generated by an uncertain signal model displayed in Fig. 1. It is assumed that Fig. 1 is an uncertain signal model for a scalar $y_{j}$. Here, $y_{j}$ is the observed value of an unknown scalar $m_{j}$ being affected by measurement

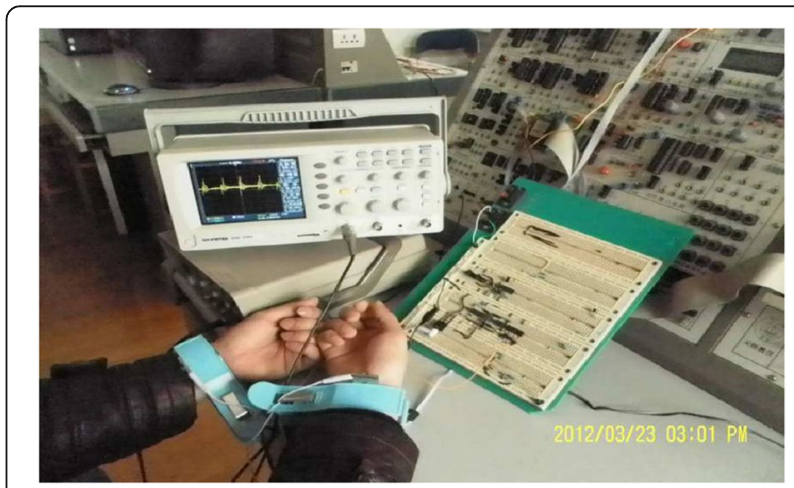

Fig. 5 ECG measurement physical diagram 


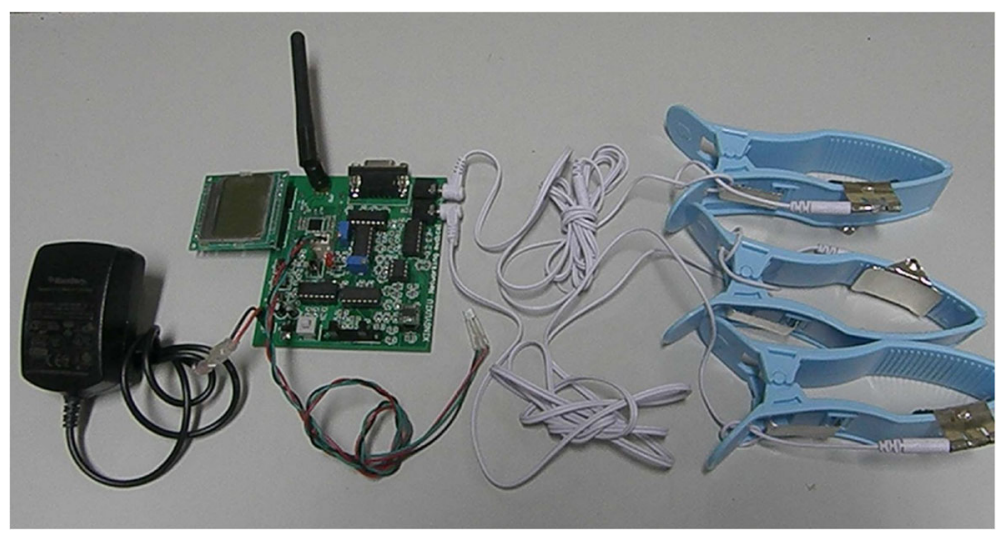

Fig. 6 Hardware node physical diagram

noise $v_{j}$ and uncertainty $u_{j}$. The uncertainty $u_{j}$ (equal to the dot product of $\mathrm{Gj} \in \mathrm{RK}$ and $\alpha \in \mathrm{RK}$ ) is being generated by a linear combination of $K$ different sources: $(\alpha 1, \cdots, \alpha \mathrm{K})$ that the $j$ th element of $y$ is generated as

$$
y_{j}=m_{j}+u_{j}+v_{j}
$$

where $v_{j}$ is the measurement noise, $u_{j}$ is the uncertainty affecting the model, and $m_{j}$ is an unobserved scalar variable. The uncertainties are assumed to be generated by linearly transforming a $\mathrm{K}$-dimensional $(\mathrm{K} \leq \mathrm{P})$ vector $\alpha=\alpha=\left[\alpha_{1} \cdots \alpha_{K}\right]^{T} \in \mathbb{R}^{K}$ as follows:

$$
\left[\begin{array}{c}
u_{1} \\
\vdots \\
u_{P}
\end{array}\right]=\left[\begin{array}{ccc}
G_{11} & \cdots & G_{1 K} \\
\vdots & & \vdots \\
G_{P 1} \cdots G_{P K}
\end{array}\right]\left[\begin{array}{c}
\alpha_{1} \\
\vdots \\
\alpha_{K}
\end{array}\right] .
$$

Defining $G_{j}=\left[G_{j 1} \cdots G_{j K}\right]^{T} \in \mathbb{R}^{K}, u_{j}$ can be expressed as the dot product of $G_{j}$ and $\alpha$, i.e.,

$$
u_{j}=\left(G_{j}\right)^{T} a
$$

Our approach is of

1. Treating all the variables as uncertain being characterized by fuzzy membership functions

2. Assuming that medical data, under the given status of a patient, is generated by a finite mixture of uncertain signal models

3. Determining the fuzzy membership functions on variables with the help of experimentally measured data samples in an analytical manner using variational optimization.

\subsection{System software design}

Software is the soul of the system. Whether the software structure is reasonable directly affects the operating efficiency of the hardware. A reasonable software structure

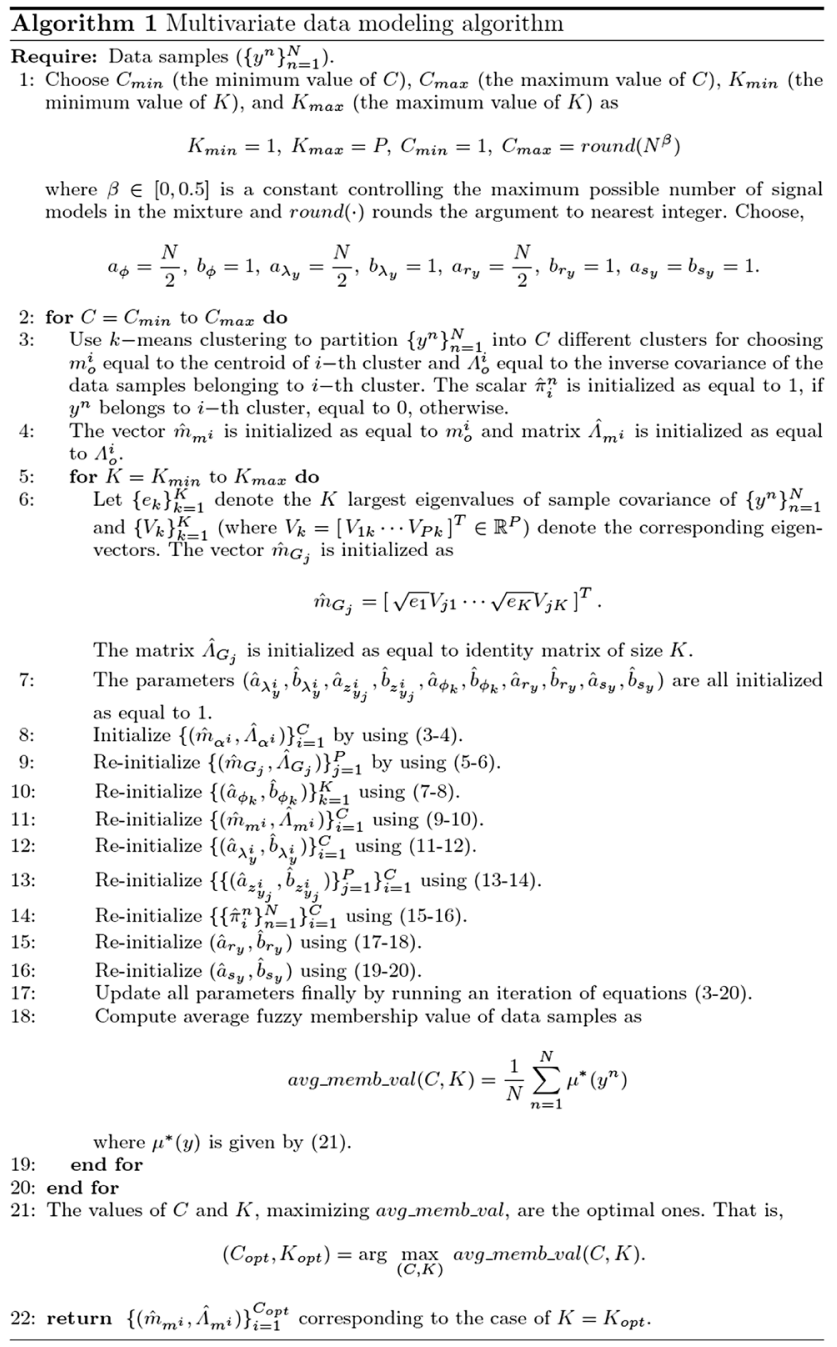

can reduce the system's electromagnetic interference, enhance the system's anti-jamming capability, and improve the reliability of the system. Based on hardware and 
software, this design writes the software program. The system software block diagram is shown in Fig. 7. The block diagram contains the functional modules of all nodes, including the data acquisition module of the sensor node, the display of the coordinator node and the serial communication module, and the wireless communication module that runs through the entire system.

The CC2430 chip integrates the 8051 core. The 8051 supports more development languages, including assembly, PL/M, BASIC, and C. In high-level languages, $C$ is a language that is relatively close to hardware. It has good portability, high hardware control capabilities, strong expression, and computing power. $\mathrm{C}$ programming is more in line with people's thinking habits. The freedom degree of program design is large, and modular programming is also available, which increases the readability and portability of the program. If the system needs to achieve the same or similar functions in subsequent development, the developer can make changes to the previous module to maximize resource sharing. Compared with assembly language, $C$ language has good portability. The $C$ language has obvious advantages over the operating system and system utilities and where the hardware needs to be operated on [10]. Programmers who use C programming will feel less restricted, more flexible, and more powerful. They can write any type of program. At the same time, the system selects IAR Embedded Workbench as the development environment. IAREWRM is an integrated development environment developed by IARsystems Company. Users can develop applications for many different target processors. IAR Embedded Workbench is suitable for a large number of 8-bit, 16-bit, and 32-bit microprocessors and microcontrollers. Therefore, users can also develop in the familiar development environment when developing new projects. It provides users with a development environment that is easy to learn and has the greatest amount of code inheritance, as well as support for most and special goals. With IAR tools, users can save time [11].

This article uses the ZigBee protocol system. Difference between Bluetooth and Zigbee Technologies has been compared in Table 1. According to the IEEE 805.12.4 standard protocol, the working frequency band of ZigBee is divided into three frequency bands. The three operating bands are relatively apart, and the number of channels in each band is different. Therefore, in this technical standard, the modulation methods and transmission rates in different frequency bands are different. They are respectively $868 \mathrm{MHz}, 915 \mathrm{MHz}$, and $2.4 \mathrm{GHz}$. The $2.4 \mathrm{GHz}$ frequency band is divided into 16 channels. This frequency band is a universal industrial, scientific, and medical frequency band. The frequency band is a toll-free, application-free radio frequency band. In this band, the data transmission rate is $250 \mathrm{kbps}$. We use this band to transmit data. In terms of networking performance, ZigBee devices can be constructed as a star network or a peer-to-peer network. In each ZigBee-based wireless network, the connection address code is divided into 16-bit address or 64-bit address. The maximum number of devices it can accommodate is 216 and 264 . Therefore, it has larger network capacity. In the wireless communication technology, a carrier sense multiple access with collision avoidance (CSMACA) method is adopted to effectively avoid collisions between radio carriers. To ensure the reliability of the transmitted data, a complete response communication protocol is established. ZigBee equipment is a low-power equipment. Its transmission output is $0 \sim 3.6 \mathrm{dBm}$, and communication distance is $30 \sim 70 \mathrm{~m}$. It has energy detection and link quality indicator capability. According to these test results, the device can automatically adjust the transmit power. Under the condition of guaranteeing the quality of the communication link, the equipment energy is minimally consumed. To ensure the security of the communication data between ZigBee devices, ZigBee technology uses a 128-bit encryption algorithm to encrypt the transmitted data information. ZigBee's architecture consists of various modules called layers. The Service-oriented architecture (SOA) has been used, each layer provides a specific service for its upper layer, that means, all data transmission services are designed in the data service entity, and all other management services are designed in the management entity.

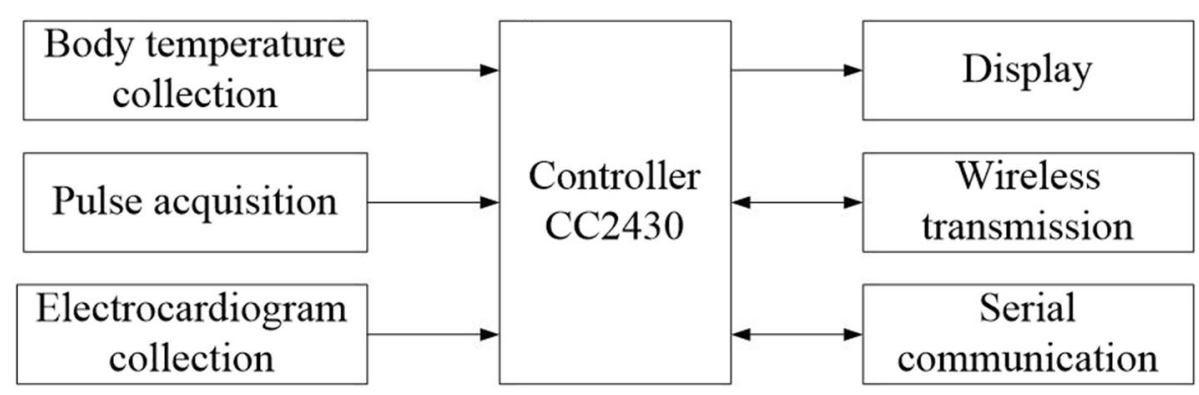

Fig. 7 System software block diagram 
Table 1 Bluetooth vs. ZigBee

\begin{tabular}{lll}
\hline & Bluetooth ( 1$)$ & ZigBee \\
\hline Protocol stack & $250 \mathrm{~kb}$ & $<32 \mathrm{~kb}(4 \mathrm{~kb})$ \\
Range & $10-100 \mathrm{~m}$ & $30-100 \mathrm{~m}$ \\
Link rate & $1 \mathrm{Mbps}$ & $250 \mathrm{kbps}$ \\
Battery & Rechargeable & Non-rechargeable \\
Devices & 8 & $2 \wedge 16$ \\
Air interface & FHSS & DSSS \\
Usage & Frequently & Infrequently \\
Network join time & Long & Short \\
Extendibility & No & Yes \\
Security & PIN, 64 bit, 128 Bit & 128 bit, AES \\
\hline
\end{tabular}

Each service entity provides its upper layer with an interface through a corresponding service access point (SAP). Each service access point completes the corresponding function through a service primitive.

Among them, P0_0 is set as general $\mathrm{I} / \mathrm{O}$ to directly collect DS18B20 temperature value. P0_1 is set to the external interruption (rising edge trigger), and the heart rate value is calculated by reading the time difference between the two interrupts. The P0_6 pin is configured as an ADC input (the corresponding bit in the ADCCFG register is set to 1 ). The 6th channel is selected with single-ended input mode and 14-bit resolution. The $1.25 \mathrm{~V}$ reference voltage is internally generated. When the ADCCON1.ST bit is set to 1 , a conversion sequence is initiated and the analog ECG voltage signal is converted to a digital quantity. At the end of the conversion, the status bit ADCCON1.ECO is set to high level. The conversion result is stored in ADCH and ADCL in the form of two complement to complete the ECG signal acquisition. There are mainly three types of nodes in the monitoring network, namely sensor nodes, routing nodes, and coordinator nodes. After the coordinator node is powered on, it is responsible for establishing and initializing the network, sending a network beacon, determining the network operating channel, and allocating a 16-bit network address. After the routing node joins the network, it receives the data sent by the sensor node and forwards the data to the coordinator node. The sensor node only sends and receives the signal and does not have the forwarding function. After powering on, it automatically initiates a bind request. After the binding with the parent (routing) node is established, the collected body temperature, pulse, and ECG data are sent to the routing node. In addition, the coordinator node sends data to the PC through the serial port. Doctors can query and analyze data through the user management interface.

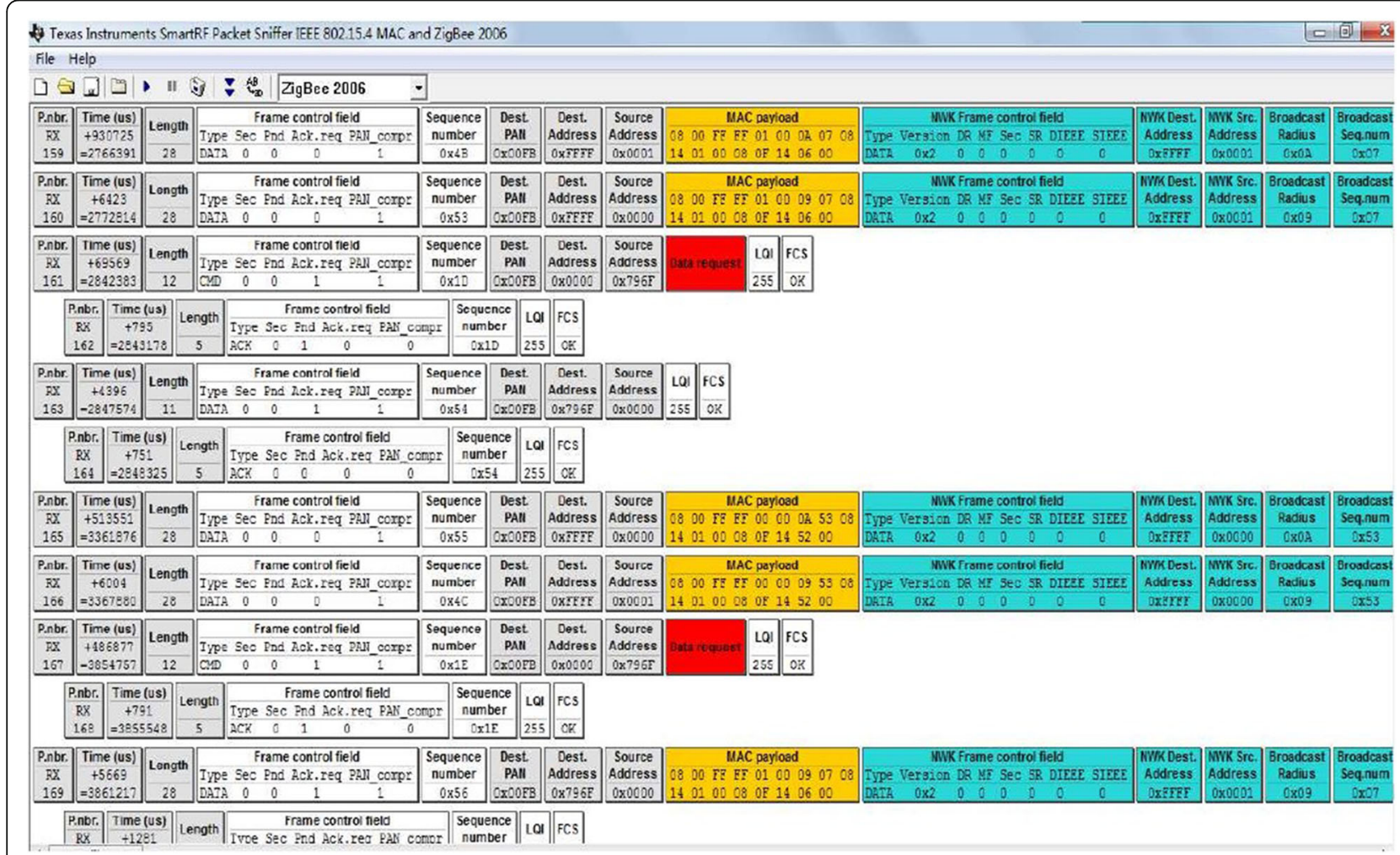

Fig. 8 ZigBee network generation interface 


\section{Results and discussion}

The network is built in the laboratory environment, and the ZigBee network test and data acquisition test are completed. The concrete realization method is as follows: The coordinator node is connected to the PC through the serial port. After the node is powered on, it automatically establishes a successful network. Then, the sensor node and the routing node are opened in turn. After all the nodes join the network, the network topology is observed. All packets in the network are captured through sniffe software. After the coordinator node is powered on, a network is established and searches for network access requests. Its source address is $0 x 0000$. After the routing node is powered on, it sends a network access request and applies for joining the network. The source address of the routing node is 0x0001, and the source address of the sensor node is $0 x 796 \mathrm{~F}$. The network is generated. As shown in Fig. 8, the long address of all the nodes that join the network, the short address assigned by the network, and the transmitted frame data information are displayed.

\section{Conclusion}

Firstly, a monitoring system for real-time remote monitoring of inpatient physiological signals is designed. The hardware circuit design of the sensor node and the coordinator node and the corresponding software program design are achieved. Among them, the sensor node can collect three physiological signals, including body temperature, pulse, and ECG. The sensor node forms a wireless network with the routing node and the coordinator node. Then, the collected physiological information is transmitted to the information management system of the upper computer for the doctor to view at any time. Finally, the hardware and software are combined to debug the system modules online. The experiment proves that the network node is reliable and the data transmission is accurate, basically meeting the design requirements of this paper. And the analytically derived expressions for fuzzy membership functions we present in this work should facilitate a system theoretic approach to mathematically design the medical expert systems.

\section{Abbreviations \\ ADC: Analog-to-digital converter; CC2430: A True System-on-Chip solution for ZigBee; ECG: Electrocardiogram; LDA: Linear discriminant analysis; QDA: Quadratic discriminant analysis; RBF: Radial basis function; RF: Radio frequency; SVM: Support vector machines}

\section{Funding}

This work was supported by the National Natural Science Foundation of China (61662045) and the Special Program of talents Development for Excellent Youth Scholars in Tianjin.

Availability of data and materials

The materials and data are true and reliable in this paper.
Authors' contributions

$Y-J Z$ is the corresponding author. Z-WP and Y-JZ conceived the proposed scheme. Z-WP and M-K conducted the detailed derivation of the proposed algorithm and carried out the most experiments and data analysis. Y-JZ and Y-JF carried out the part experiments and data analysis. Y-JF helped to improve the experimental simulation. All authors have read and approved the final manuscript.

\section{Competing interests}

The authors declare that they have no competing interests.

\section{Publisher's Note}

Springer Nature remains neutral with regard to jurisdictional claims in published maps and institutional affiliations.

\section{Author details}

'Department of Electronic Information Engineering, Nanchang University, Nanchang, China. ${ }^{2}$ Binhai Industrial Technology Research Institute of Zhejiang University, Tianjin, China. ${ }^{3}$ Faculty of Computer Science and Electrical Engineering, University of Rostock, Rostock, Germany.

Received: 5 May 2018 Accepted: 11 June 2018

Published online: 11 July 2018

\section{References}

1. W Zhang, K Thurow, R Stoll, A knowledge-based telemonitoring platform for application in remote healthcare. Int J Comput Commun Control 9(5), 644-654 (2014)

2. R Piyare, Internet of things: ubiquitous home control and monitoring system using android based smart phone. Int J Internet Things 2(1), 5-11 (2013)

3. J Jiao, $\mathrm{H} \mathrm{Ma}$, Y Qiao, et al., Design of farm environmental monitoring system based on the Internet of Things. Adv J Food Sci Technol 6(3), 368-373 (2014)

4. JH Abawajy, MM Hassan, Federated internet of things and cloud computing pervasive patient health monitoring system. IEEE Commun Mag 55(1), 4853 (2017)

5. G Marques, P Rui, An indoor monitoring system for ambient assisted living based on internet of things architecture. Int J Environ Res Public Health 1(11), 1152 (2016)

6. VP Rachim, Y Jiang, HS Lee, WY Chung, Demonstration of long-distance hazard-free wearable eeg monitoring system using mobile phone visible light communication. Opt Express 25(2), 713-719 (2017)

7. R Prakash, Australian journal of Basic and applied sciences internet of things (IoT) enabled wireless patient monitoring system using CC3200. Aust J Basic Appl Sci 9(916), 275-281 (2015)

8. HS Kim, JS Seo, J Seo, A daily activity monitoring system for internet of things-assisted living in home area networks. Kor J Pathol 6(1), 399 (2016)

9. W Zhang, J Yang, Y Fang, H Chen, Y Mao, M Kumar, Analytical fuzzy approach to biological data analysis. Saudi J Biol Sci 24(3), 563-573 (2017)

10. S Wu, A traffic motion object extraction algorithm. Int J Bifurcation Chaos 25(14), Article Number 1540039 (2015)

11. L Gui, TY Ruan, ZZ Wang, et al., CNC online monitoring system based on internet of things. Adv Mat Res 1079-1080(6), 672-678 (2015)

\section{Submit your manuscript to a SpringerOpen ${ }^{\mathcal{O}}$ journal and benefit from:}

- Convenient online submission

- Rigorous peer review

- Open access: articles freely available online

- High visibility within the field

- Retaining the copyright to your article

Submit your next manuscript at $>$ springeropen.com 